\section{Covariance Averaging in the Analysis of Uncertain Systems}

Steven R. Hall, Douglas G. MacMartin, and Dennis S. Bernstein

Abstract-The effects of parametric uncertainty in stable state space systems are anslyzed by averaging the state covariance over the statistic of the uncertain parameters. For natural frequency uncertainty, this computation is related to the Fourier transform of the probability density function of the uncertain parameter. Equipartition and incis density function of the uncertain parameter. Equipartition and incoherde oscillator. Averaging over discrete uncertainty model yields the Bourret design equations, while averaging over a Cauchy uncertainty distribution yields the "maximum entropy" covariance equation of Hyland.

\section{INTRODUCTION}

In recent years, there has been significant progress in analysis and compensator synthesis for systems with unstructured uncertainty. However, problems with parametric uncertainty are much less well understood. The goal of this note is to examine the effects of parametric uncertainty in stable state space systems by averaging the state covariance with respect to a stochastic parametric uncertainty description. The $\mathscr{H}_{2}$ cost of a known system can be computed from the system covariance; similarly, the expected cost for an uncertain system can be computed from the average covariance. Previous research on cost or covariance averaging for control design includes [1] and [2, p. 114]

To simplify the analysis, attention is confined to systems with eigenvalue uncertainty only. This restriction is less significant than one might think; performance in structural control problems is often limited by uncertainty in the natural frequencies, rather than uncertainty in the residues or zero locations that result from eigenvector perturbations (e.g., [3], [4].) The key insight is that for modal frequency uncertainty, the average covariance is related to the Fourier transform of the probability density function of the uncertain parameters. The covariance averaging techniques are then applied to a single mode oscillator example to demonstrate the statistical phenomena of incoherence (modal decorrelation) and equipartition (modal energy equilibration). These are several of the fundamental assumptions of Statistical Energy Analysis (SEA) [5], a field that uses a stochastic approach to analyze the response of uncertain flexible structures.

Explicit solutions for the average covariance in terms of Lyapunov-like equations are obtained for several probability distributions. A discrete distribution leads to the Bourret approximation [1] to the average cost over a uniform distribu-

Manuscript received September 26, 1991; revised April 14, 1992 and August 3, 1992. This work was supported by the Sandia National Laboratory under contract 69-4391, the MIT Space Engineering Research Center under NASA grant NAGW-1335, and by the Air Force Office of Scientific Research under contract F49620-89-C-0011.

S. R. Hall is with the Space Engineering Research Center, Department of Aeronautics and Astronautics, Massachusetts Institute of Technology, Cambridge, MA 02139

D. G. MacMartin was with the Space Engineering Research Center, Department of Aeronautics and Astronautics, Massachusetts Institute of Technology, Cambridge, MA 02139. He is currently with the Institute for Aerospace Research, National Research Council of Canada, Ottawa, Aerospace Research, Nation
Ontario, Canada K1A ORG.

Ontario, Canada K1A ORG.
D. S. Bernstein is with the Department of Aerospace Engineering, The University of Michigan, Ann Arbor, MI 48109-2140.

IEEE Log Number 9212879. tion. Averaging over a Cauchy distribution yields the covariance equation of the "maximum entropy" robustness technique [6], which was originally justified by means of a multiplicative white noise model (e.g., [7].) The covariance of the state that satisfies a particular differential equation with multiplicative white noise is the same as the average covariance for a state that satisfies a differential equation where the coefficients are constant, but uncertain with known distribution.

One difficulty with applying many norm-based robust control design approaches to structural control problems is that the phase information in the parametric uncertainty of the structure is important. The structure approximately conserves energy, although the modal frequencies may be highly uncertain. Thus, because the Bourret and maximum entropy approaches evaluate the cost from the average covariance over a set of conservative systems, both implicitly use and preserve the knowledge that the structure is conservative.

\section{Average Covariance}

Consider the state space system

$$
\dot{x}=A x+w \quad x(0)=x_{0}
$$

for $x \in \mathbb{R}^{n}$, and white driving noise $w$. Assume that uncertainty in $A$ is represented by

$$
A=A_{0}+\sum_{i=0}^{r} \sigma_{i} A_{i}
$$

where the uncertain parameters $\sigma_{i}$ have known joint probability density function $p\left(\sigma_{1}, \cdots, \sigma_{r}\right)$, and the given matrices $A_{i}$ describe the effect of each uncertain parameter. For simplicity, the following development will concentrate on the case of a single uncertain parameter, $\sigma=\sigma_{1}$. The uncertainty structure $A_{1}$ and the original system matrix $A_{0}$ will be required to commute. In general, $A_{1}$ and $\boldsymbol{A}_{0}$ commute if and only if both are simultaneously diagonalizable by the same eigenvector matrix; hence the uncertainty can change the eigenvalues, but not the eigenvectors (or mode shapes) of the original system.

The covariance matrix $Q(t) \triangleq\left\langle x(t) x^{T}(t)\right\rangle_{w}$ associated with the system in (1) is given by

$$
\dot{Q}=A Q+Q A^{T}+V \quad Q(0)=Q_{0}=x_{0} x_{0}^{T}
$$

where the constant matrix $V$ is the intensity of the white noise $w$, and $\langle\cdot\rangle_{w}$ denotes expectation over $w$. Equation (3) can be solved explicitly using Kronecker algebra [8]. The vector obtained by stacking the columns of $Q$ is denoted by vec $\{Q\}$. Similarly, define $q_{0} \triangleq \operatorname{vec}\left\{Q_{0}\right\}$, and $v \triangleq \operatorname{vec}\{V\}$. The symbols $\otimes$ and $\oplus$ denote, respectively, the Kronecker product and sum operators. Then (3) can be written as follows:

$$
\dot{q}=(A \oplus A) q+v \quad q(0)=q_{0}
$$

and thus

$$
q(t)=e^{(A \oplus A) t} q_{0}+\int_{0}^{t} e^{(A \oplus A)(t-\tau)} v d \tau .
$$

The following facts are required to proceed further.

Lemma 1:

$$
\text { i) } \begin{aligned}
& A=A_{0}+\sigma_{1} A_{1} \\
& \text { ii) } \begin{aligned}
A_{0} A_{1} & =A_{1} A_{0} \\
& \Rightarrow\left(A_{0} \oplus A_{0}\right)\left(A_{1} \oplus A_{1}\right)=\left(A_{1} \oplus A_{1}\right)\left(A_{0} \oplus A_{0}\right) .
\end{aligned}
\end{aligned}
$$


iii) If $A_{1}=\Psi \Lambda \Psi^{-1}$ is diagonalizable, with $\Lambda=\operatorname{diag}\left\{\lambda_{i}\right\}$, then

$$
\begin{aligned}
& A_{1} \otimes A_{1}=(\Psi \otimes \Psi)(\Lambda \otimes \Lambda)(\Psi \otimes \Psi)^{-1} \\
& A_{1} \oplus A_{1}=(\Psi \otimes \Psi)(\Lambda \oplus \Lambda)(\Psi \otimes \Psi)^{-1} .
\end{aligned}
$$$$
\text { iv) } e^{(X+Y) t}=e^{X t} e^{Y t} \forall t \Leftrightarrow X Y=Y X \text {. }
$$

Proof: Results i)-iii) follow from the definitions of the Kronecker operators [8]. The final assertion is from [9, p. 171].

Now define

$$
\mathscr{Q}(t) \triangleq e^{\left(A_{0} \oplus A_{0}\right) t}\left(\int_{-\infty}^{\infty} e^{\sigma\left(A_{1} \oplus A_{1}\right) t} p(\sigma) d \sigma\right) .
$$

Then from Lemma 1 the average covariance is given by

$$
\langle q(t)\rangle_{\sigma}=\operatorname{vec}\left\{\langle Q(t)\rangle_{\sigma}\right\}=\mathscr{Q}(t) q_{0}+\int_{0}^{t} \mathscr{Q}(t-\tau) v d \tau
$$

This involves an expectation over both the uncertainty and the driving noise. The assumption that $A_{0}$ and $A_{1}$ commute is required by Lemma 1-iv). If $A_{1}=\Psi \Lambda \Phi^{T}$ is diagonalizable with $\Lambda=\operatorname{diag}\left\{\lambda_{i}\right\}, \Psi=\left[\begin{array}{lll}\psi_{1} & \cdots & \psi_{n}\end{array}\right], \boldsymbol{\Phi}=\left[\begin{array}{lll}\phi_{1} & \cdots & \phi_{n}\end{array}\right]$, then (6) can be written as follows:

$$
\begin{aligned}
& \mathscr{Q}(t)=e^{\left(A_{0} \oplus A_{0}\right) t} \sum_{i=1}^{n} \sum_{j=1}^{n}\left(\psi_{i} \otimes \psi_{j}\right) \\
& \quad \cdot\left(\phi_{i}^{T} \otimes \phi_{j}^{T}\right) \int_{-\infty}^{\infty} e^{\sigma\left(\lambda_{i}+\lambda_{j}\right) t} p(\sigma) d \sigma .
\end{aligned}
$$

If only the modal frequencies of the system are uncertain, then the eigenvalues $\lambda_{i}$ of $A_{1}$ are purely imaginary, and hence the integrals in (8) are the Fourier transforms of $p\left(\sigma /\left|\lambda_{i}+\lambda_{j}\right|\right)$.

Remark 2: For modal frequency uncertainty, the average covariance can be evaluated in terms of the Fourier transform of the probability density function.

Multiple uncorrelated uncertain parameters can be treated if $A_{i} A_{j}=A_{j} A_{i} \forall i, j \geq 0$. Additional uncertain parameters simply result in additional product terms in (6).

\section{Single MODE OSCILlator}

Of particular interest for understanding the effect of uncertainty in structures is whether the incoherence and equipartition assumptions of SEA [5] follow from averaging over uncertainty.

Definition 3: Equipartition is said to occur at time $t$ if the average energy in each state at time $t$ is the same. Incoherence is said to occur if the average cross-correlation between the state coordinates is zero. Steady-state equipartition or incoherence is said to occur if equipartition or incoherence are satisfied in the limit as $t \rightarrow \infty$.

To simplify the analysis, consider the case of a single mode oscillator. Define

$$
J \triangleq\left[\begin{array}{rr}
0 & 1 \\
-1 & 0
\end{array}\right]
$$

so that $J^{2}=-I$ and $J^{T}=-J$. Then for $\eta \geq 0$, consider the system

$$
\begin{aligned}
A & =A_{0}+\sigma A_{1} \\
A_{0} & =-\eta I+\omega J \quad A_{1}=J .
\end{aligned}
$$

The eigenvalues of this system are at $-\eta \pm j(\omega+\sigma)$, and the eigenvectors are independent of $\sigma$. In this state space basis, each element of the state vector corresponds to a normalized energy variable. Thus, if the system represents a mechanical oscillator, $x_{1}^{2}(t) / 2$ and $x_{2}^{2}(t) / 2$ are the instantaneous kinetic and potential energy. With $\langle Q(t)\rangle_{\sigma}$ as the average covariance of the system in (10), equipartition holds if $\left\langle Q_{11}(t)\right\rangle_{\sigma}=\left\langle Q_{22}(t)\right\rangle_{\sigma}$, while incoherence holds if $\left\langle Q_{12}(t)\right\rangle_{\sigma}=0$. For an undamped system with $\sigma$ fixed, the energy continually oscillates between $Q_{11}$ and $Q_{22}$, and steady-state equipartition does not occur. Similarly, the state coordinates remain correlated, and steadystate incoherence does not occur.

Rather than performing the eigen-decomposition indicated by (8), complex algebra can be avoided by noting the decomposition

$$
J \oplus J=\Phi\left[\begin{array}{cc}
0 & 0 \\
0 & 2 J
\end{array}\right] \Phi^{T} \quad A_{0} \oplus A_{0}=\Phi\left[\begin{array}{cc}
-2 \eta I & 0 \\
0 & 2 A_{0}
\end{array}\right] \Phi^{T}
$$

where the orthogonal transformation $\Phi$ is given by

$$
\Phi \triangleq \frac{1}{\sqrt{2}}\left[\begin{array}{r:r}
I & I \\
-J & J
\end{array}\right]=\left[\begin{array}{ll}
\Phi_{1} & \Phi_{2}
\end{array}\right]
$$

Using (11), it follows that

$$
\int_{-\infty}^{\infty} e^{\sigma\left(A_{1} \oplus A_{1}\right) t} p(\sigma) d \sigma=\Phi_{1} \Phi_{1}^{T}+\Phi_{2}\left(\int_{-\infty}^{\infty} e^{2 \sigma J t} p(\sigma) d \sigma\right) \Phi_{2}^{T} .
$$

Note that, analogous to Euler's formula for the scalar case, $e^{\sigma J t}=(\cos \sigma t) I+(\sin \sigma t) J$. Hence, (13) can be written in terms of the Fourier cosine and sine transforms of $p(\sigma / 2)$ :

$$
\begin{aligned}
& f_{c}(t) \triangleq \int_{-\infty}^{\infty} \cos (2 \sigma t) p(\sigma) d \sigma \\
& f_{s}(t) \triangleq \int_{-\infty}^{\infty} \sin (2 \sigma t) p(\sigma) d \sigma
\end{aligned}
$$

Consider first the average covariance for the unforced case $(V=0)$.

Theorem 4: The average covariance of the unforced single mode oscillator in (10) satisfies both steady state equipartition and incoherence if the integral of $p(\sigma)$ is absolutely continuous. Furthermore, the average total energy decays at the same rate as the energy of the nominal system.

Proof: Define $\xi(t)=\Phi^{T}\langle q(t)\rangle_{\sigma}$, so that $\xi_{1}=\left\langle Q_{11}\right\rangle_{\sigma}+$ $\left\langle Q_{22}\right\rangle_{\sigma}, \quad \xi_{2}=\left\langle Q_{21}\right\rangle_{\sigma}-\left\langle Q_{12}\right\rangle_{\sigma}, \quad \xi_{3}=\left\langle Q_{11}\right\rangle_{\sigma}-\left\langle Q_{22}\right\rangle_{\sigma}$, and $\xi_{4}=\left\langle Q_{12}\right\rangle_{\sigma}+\left\langle Q_{21}\right\rangle_{\sigma}$. Then using (11) and (13), (7) for the average covariance can be written in terms of $\xi_{i}$ as

$$
\begin{aligned}
\xi_{1}(t)= & e^{-2 \eta t} \xi_{1}(0) \\
\xi_{2}(t)= & e^{-2 \eta t} \xi_{2}(0) \\
\left(\begin{array}{l}
\xi_{3}(t) \\
\xi_{4}(t)
\end{array}\right)= & e^{-2 \eta t}\left[\begin{array}{rr}
\cos (2 \omega t) & \sin (2 \omega t) \\
-\sin (2 \omega t) & \cos (2 \omega t)
\end{array}\right] \\
& \cdot\left[\begin{array}{rr}
f_{c}(t) & f_{s}(t) \\
-f_{s}(t) & f_{c}(t)
\end{array}\right]\left(\begin{array}{l}
\xi_{3}(0) \\
\xi_{4}(0)
\end{array}\right) .
\end{aligned}
$$

In the state space basis being used, $Q_{i i}$ is the energy associated with the state $x_{i}$. Hence, $\xi_{1}$ is the average total energy of the system, and the final conclusion is immediate from (15). Equation (16) implies that $\langle Q(t)\rangle_{\sigma}$ is symmetric. Steady-state equipartition and incoherence occur if $\lim _{t \rightarrow \infty} \xi_{3}(t)=0$ and $\lim _{t \rightarrow \infty} \xi_{4}(t)=0$, which requires that in the undamped case, from $(17), \lim _{t \rightarrow \infty} f_{c}(t)=0$ and $\lim _{t \rightarrow \infty} f_{s}(t)=0$. From the Riemann-Lebesgue Lemma [10], a sufficient condition for this is that the associated measure given by $d \mu=p(\sigma) d \sigma$ is absolutely continuous. 
Remark 5: If there is a finite probability of a specific $\sigma$ being achicved (a multiple-model uncertainty), then the Fourier transform of $p$ does not tend to zero, and steady-state equipartition and incoherence do not occur.

Now consider the steady state forced response, $V \neq 0$. Defining $\nu=\Phi^{T} v$, then

$$
\begin{aligned}
\lim _{t \rightarrow \infty}\left(\begin{array}{l}
\xi_{3}(t) \\
\xi_{4}(t)
\end{array}\right)= & \int_{0}^{\infty} e^{-2 \eta t}\left[\begin{array}{rr}
\cos (2 \omega t) & \sin (2 \omega t) \\
-\sin (2 \omega t) & \cos (2 \omega t)
\end{array}\right] \\
& \cdot\left[\begin{array}{rr}
f_{c}(t) & f_{s}(t) \\
-f_{s}(t) & f_{c}(t)
\end{array}\right]\left(\begin{array}{l}
\nu_{3} \\
\nu_{4}
\end{array}\right) d t \\
= & \int_{-\infty}^{\infty} p(\sigma)\left[\begin{array}{ll}
f_{1} & f_{2} \\
f_{2} & f_{1}
\end{array}\right]\left(\begin{array}{l}
\nu_{3} \\
\nu_{4}
\end{array}\right) d \sigma
\end{aligned}
$$

where $\nu_{3}$ and $\nu_{4}$ are the third and fourth components of $\nu$ and

$$
f_{1} \triangleq \frac{\eta}{\eta^{2}+(\omega+\sigma)^{2}} \quad f_{2} \triangleq \frac{\omega+\sigma}{\eta^{2}+(\omega+\sigma)^{2}} .
$$

Both $\left|f_{1}\right|$ and $\left|f_{2}\right|$ are bounded by $1 / \eta$, and also, $\forall \epsilon>0 \exists \Delta>0$ such that $\left|f_{1}\right|<\epsilon$ and $\left|f_{2}\right|<\epsilon \forall|\sigma|>\Delta$. If $p(\sigma)$ satisfies the conditions of Theorem 4 , then $p$ can be parameterized by a scaling on the uncertainty such that $\forall \epsilon_{2}>0 \quad \exists k>0$ such that $k p(k \sigma)<\epsilon_{2} \forall|\sigma|<\Delta$, and hence $\lim _{t \rightarrow \infty} \xi_{3}(t)$ and $\lim _{t \rightarrow \infty} \xi_{4}(t)$ can be made arbitrarily small. Hence, for any probability density with a continuous integral, equipartition and incoherence for the forced case will be achieved in the limit as the uncertainty level of the probability density is increased.

\section{EXAMPLES}

For several probability distributions for the uncertainty, the average covariance can be computed via Lyapunov-like matrix equations similar to (3). Note that

$$
\begin{aligned}
\Phi_{1} \Phi_{1}^{T} & =\frac{1}{2}(I+J \otimes J) \\
\Phi_{2} \Phi_{2}^{T} & =\frac{1}{2}(I-J \otimes J) \\
\Phi_{2} J \Phi_{2}^{T} & =\frac{1}{2} J \oplus J .
\end{aligned}
$$

It follows that the general solution for $\langle q(t)\rangle_{\sigma}$ with nonzero forcing $v$ is given by (7) where, from (6) and (13),

$\mathcal{Q}(t)=\frac{1}{2} e^{\left(A_{0} \oplus A_{0}\right) t}\left[(I+J \otimes J)+(I-J \otimes J) f_{c}(t)\right.$

$$
\left.+(J \oplus J) f_{s}(t)\right]
$$

For convenience, denote the average covariance by $Q_{a}(t) \triangleq$ $\langle Q(t)\rangle_{\sigma}$, and $q_{a}(t) \triangleq \operatorname{vec}\left\{Q_{a}(t)\right\}$. The following identities will also be useful:

$$
\begin{aligned}
(J \oplus J)^{2} & =-2(I-J \otimes J) \\
(J \oplus J)(J \otimes J) & =-(J \oplus J) \\
(I-J \otimes J)^{2} & =2(I-J \otimes J) \\
(I-J \otimes J)(I+J \otimes J) & =0 .
\end{aligned}
$$

First, consider the case involving only two possible values, $\sigma= \pm \Delta$, for the uncertain parameter. This is akin to a multiple model description of uncertainty.

Theorem 6 (Discrete Uncertainty): Consider the system in (10). If the probability density function for $\sigma$ is given by

$$
p(\sigma)=\frac{1}{2} \delta(\sigma-\Delta)+\frac{1}{2} \delta(\sigma+\Delta)
$$

where $\delta$ is the Dirac delta function, then the average covariance $Q_{a}(t)$ is the solution to

$$
\begin{aligned}
& \dot{Q}_{a}=A_{0} Q_{a}+Q_{a} A_{0}^{T}+\Delta\left(A_{1} Q_{b}+Q_{b} A_{1}^{T}\right)+V \\
& \dot{Q}_{b}=A_{0} Q_{b}+Q_{b} A_{0}^{T}+\Delta\left(A_{1} Q_{a}+Q_{a} A_{1}^{T}\right)
\end{aligned}
$$

with initial conditions $Q_{a}(0)=Q_{0}$ and $Q_{b}(0)=0$.

Proof: For this distribution, $f_{c}(t)=\cos (2 \Delta t)$ and $f_{s}(t)=0$, from which the average covariance is given by (7) and (21). Differentiating $q_{a}(t)$ yields

$$
\begin{aligned}
& \dot{q}_{a}(t)=\left(A_{0} \oplus A_{0}\right) q_{a}(t)-\Delta e^{\left(A_{0} \oplus A_{0}\right) t} \\
& \cdot(I-J \otimes J) \sin (2 \Delta t) q_{0}+v \\
& -\int_{0}^{t} \Delta e^{\left(A_{0} \oplus A_{0}\right)(t-\tau)}(I-J \times J) \\
& \sin (2 \Delta(t-\tau)) v d \tau
\end{aligned}
$$

Define the auxiliary variable $q_{b}(t)=\operatorname{vec}\left\{Q_{b}(t)\right\}$ by

$$
\begin{aligned}
q_{b}(t)= & \frac{1}{2} e^{\left(A_{0} \oplus A_{0}\right) t}(J \oplus J) \sin (2 \Delta t) q_{0} \\
& +\frac{1}{2} \int_{0}^{t} e^{\left(A_{0} \oplus A_{0}\right)(t-\tau)}(J \oplus J) \sin (2 \Delta(t-\tau)) v d \tau .
\end{aligned}
$$

Using (23), one can therefore write

$$
\dot{q}_{a}(t)=\left(A_{0} \oplus A_{0}\right) q_{a}(t)+\Delta(J \oplus J) q_{b}(t)+v
$$

with the initial condition $q_{a}(0)=q_{0}$. Similarly, $\dot{q}_{b}(t)$ can be expressed in terms of $q_{a}(t)$ and $q_{b}(t)$ using (22), with $q_{b}(0)=0$ from (30). The result follows immediately.

Remark 7: Equations (27) and (28) are precisely the equations obtained in [1] for the Bourret approximation to the average covariance for a uniform distribution.

The conclusion of Theorem 6 also applies to arbitrary $A_{0}$ and $A_{1}$; they may have arbitrary dimension, and need not commute. Denote the covariances corresponding to the two possible models, $A=A_{0} \pm \Delta A_{1}$, by $Q_{1}$ and $Q_{2}$. Then $Q_{a}=(1 / 2)\left(Q_{1}+Q_{2}\right)$ and $Q_{b}=(1 / 2)\left(Q_{1}-Q_{2}\right)$; adding and subtracting the Lyapunov equations solved by $Q_{1}$ and $Q_{2}$ yields (27) and (28).

Theorem 8 (Cauchy Distribution): Consider the system in (10). If the probability density function for $\sigma$ is given by

$$
p(\sigma)=\frac{\Delta / \pi}{\sigma^{2}+\Delta^{2}}
$$

with $\Delta>0$, then the average covariance is the solution to

$$
\begin{aligned}
\dot{Q}_{a}=\left(A_{0}+\frac{1}{2} \Delta A_{1}^{2}\right) Q_{a} & +Q_{a}\left(A_{0}+\frac{1}{2} \Delta A_{1}^{2}\right)^{T} \\
& +\Delta A_{1} Q_{a} A_{1}^{T}+V
\end{aligned}
$$

with initial condition $Q_{a}(0)=Q_{0}$

Proof: For this distribution, $f_{c}(t)=e^{-2 \Delta|t|}$ and $f_{s}(t)=0$, from which the average covariance is given by (7) and (21). For $t \geq 0$, differentiating $q_{a}(t)$, and using (24) and (25) yields

$$
\dot{q}_{a}(t)=\left(A_{0} \oplus A_{0}\right) q_{a}(t)-\Delta(I-J \otimes J) q_{a}(t)+v \text {. (34) }
$$

The conclusion is obtained by noting that $I=-A_{1}^{2}$.

Remark 9: Equation (33) is precisely the covariance equation in the maximum entropy design equations $[11,(212)]$, for a single uncertain parameter.

The covariance of the state which satisfies (1) and (10) where $\sigma$ has a Cauchy distribution given by (32), is precisely the same as the covariance of the state that satisfies a differential equation of the same form, but where $\sigma$ is replaced by a white noise 
process of intensity $\sqrt{\Delta}$. This is a powerful result, as it relates two apparently different approaches, and demonstrates that the maximum entropy approach [6] can be interpreted as a cost averaging approach.

The behavior of $\left\langle Q_{11}\right\rangle_{\sigma}$ and $\left\langle Q_{22}\right\rangle_{\sigma}$ with various probability distributions is shown in Fig. 1, starting from an initial condition where all the energy is in the first state. Equipartition occurs for a uniform and Cauchy distribution, but not for the discrete uncertainty distribution, since $\lim _{t \rightarrow \infty} f_{c}(t) \neq 0$ for that case. The cross-correlation between the two state variables demonstrates a similar conclusion for incoherence.

Theorem 8 demonstrates that the average covariance for a Cauchy distribution can be computed by solving a single Lyapunov equation. More generally, it is possible to compute the average covariance for any rational and proper distribution from a set of coupled Lyapunov equations.

Theorem 10 (Rational Distribution): Let $g(s)=c(s I-\mathscr{A})^{-1} b$ be positive real, where $\mathscr{A}$ is an arbitrary asymptotically stable $n \times n$ matrix with elements $a_{i j}$, and $b, c^{T}$ are of dimension $n \times 1$, satisfying $c b=1$. Consider the system in (10). If the probability density function for $\sigma$ satisfies

$$
p(\sigma)=\frac{1}{2 \pi}\left(g(j \sigma)+g^{*}(j \sigma)\right)
$$

then the average covariance satisfies $Q_{a}(t)=\sum_{i=1}^{n} c_{i} Q_{i}(t)$, where the $Q_{i}(t)$ solve

$$
\dot{Q}_{i}=A_{0} Q_{i}+Q_{i} A_{0}^{T}+\sum_{j=1}^{n} a_{i j}\left(Q_{j}-J Q_{j} J^{T}\right)+b_{i} V
$$

for $i=1, \cdots, n$, with initial conditions $Q_{i}(0)=b_{i} Q_{0}$.

Proof: First note that $p(\sigma)>0$ since $g(s)$ is positive real. For this distribution, $f_{c}(t)=c e^{2 . \alpha / k} \mid b$ and $f_{s}(t)=0$. For $p(\sigma)$ to be a probability density function, $f_{c}(0)=\int_{-\infty}^{\infty} p(\sigma) d \sigma=1$, and hence the condition $c b=1$ is required.

With $b_{i}$ denoting the $i$ th component of the vector $b$, and $\left[e^{2 . x t} b\right]_{i}$ as the $i$ th component of the vector $e^{2 . x t} b$, then for $i=1, \cdots, n$ define

$$
\begin{aligned}
q_{i}(t) \triangleq & \frac{1}{2} e^{\left(A _ { 0 } ^ { \oplus \oplus A _ { 0 } ) t } \left\{(I+J \otimes J) b_{i}\right.\right.} \\
& \left.+(I-J \otimes J)\left[e^{2 \otimes t} b\right]_{i}\right\} q_{0} \\
& +\frac{1}{2} \int_{0}^{t} e^{\left(A_{0}^{\oplus A_{0}}\right)(t-\tau)}\left\{(I+J \otimes J) b_{i}\right. \\
& \left.+(I-J \otimes J)\left[e^{2 \otimes(t-\tau)} b\right]_{i}\right\} v d \tau
\end{aligned}
$$

with the initial conditions $q_{i}(0)=b_{i} q_{0}$. With this definition, then from (7) and (21), $q_{a}(t)=\sum_{i=1}^{n} c_{i} q_{i}(t)$ for $t \geq 0$, since $\sum_{i=1}^{n}$ $c_{i} b_{i}=1$. Differentiating (37) for each $i$ yields

$$
\dot{q}_{i}(t)=\left(A_{0} \oplus A_{0}\right) q_{i}(t)+\sum_{j=1}^{n} a_{i j}(I-J \otimes J) q_{j}(t)+b_{i} \nu
$$

The conclusion follows.

Remark 11: The Cauchy distribution in Theorem 8 corresponds to $\mathscr{A}=-\Delta, b=c=1$.

Now consider a two mode system, where

$$
A_{0}=\left[\begin{array}{cc}
-\eta_{1} I+\omega_{1} J & 0 \\
0 & -\eta_{2} I+\omega_{2} J
\end{array}\right] \quad A_{1}=\left[\begin{array}{cc}
\lambda_{1} J & 0 \\
0 & \lambda_{2} J
\end{array}\right]
$$
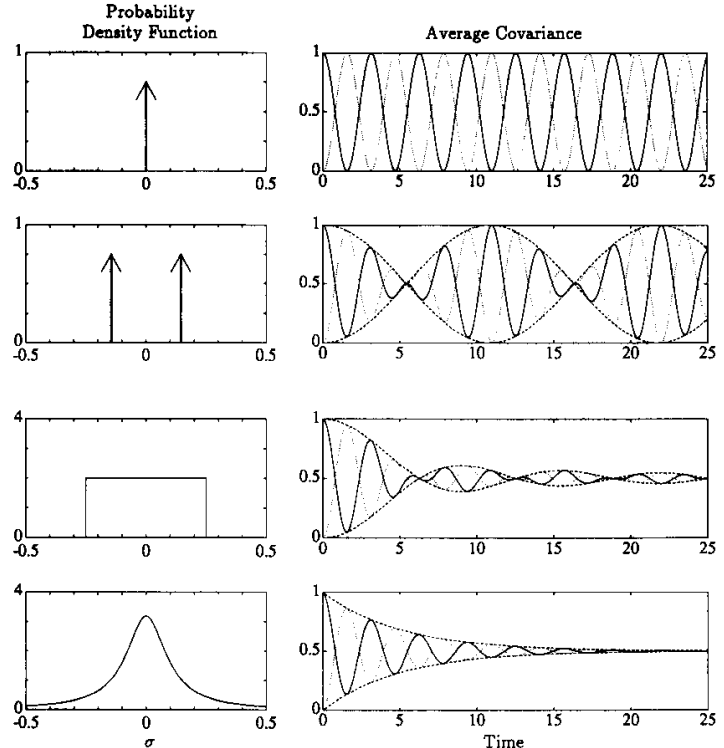

Fig. 1. Equipartition behavior: average unforced covariance from initial conditions (right) and corresponding probability density function (left). On right, $\left\langle Q_{11}\right\rangle_{\sigma}$ (solid), $\left\langle Q_{22}\right\rangle_{\sigma}$ (dotted), and the envelope of the average (dashed). From top, nominal case, discrete uncertainty, uniform distribution, and Cauchy distribution of uncertainty.

and $\lambda_{1}$ and $\lambda_{2}$ are arbitrary real numbers. The primary question of interest is whether incoherence and equipartition among different modes arises from averaging over uncertainty.

Theorem 12: The average covariance of the system $\dot{x}=$ $\left(A_{0}+\sigma A_{1}\right) x$ described by (39) satisfies steady state incoherence between modes, provided $d \mu=p(\sigma) d \sigma$ is absolutely continuous and $\left|\lambda_{1}\right| \neq\left|\lambda_{2}\right|$.

Proof: The proof is similar to that of Theorem 4. In a similar fashion to (11), write $A_{1} \oplus A_{1}=\Phi \Lambda \Phi^{T}$ where $\Lambda$ has eigenvalues $\left((-1)^{\lambda} \lambda_{i}+(-1)^{m} \lambda_{k}\right) j$ for $i, k, l, m=1,2$, and $\Phi$ is an orthogonal transformation. The zero eigenvalues $(l \neq m$, $i=k$ ) correspond to conservation of energy, and symmetry. The conclusion that every off-diagonal element of $\langle Q(t)\rangle_{\sigma}$ decays with time follows from examining the remaining eigenvectors, which have nonzero eigenvalues if $\left|\lambda_{1}\right| \neq\left|\lambda_{2}\right|$. As before, $\lim _{t \rightarrow x} f_{c}(t)=0$ and $\lim _{t \rightarrow \infty} f_{s}(t)=0$ are required.

In general, the average correlation between the states associated with different modes tends to zero in the unforced case. Conclusions in the forced case are similar to the conclusions for the single mode forced case.

If the average covariance is finite, then the system must be stable at almost every value of the uncertain parameter [1]. This suggests that a covariance averaging approach based on the results presented herein could be used for robust control synthesis. However, even if the uncertainty structure commutes with the open-loop system matrix, it will not, in general, commute with the closed-loop system matrix. If, however, the closedloop eigenvectors are close to the open-loop eigenvectors, then the errors incurred by assuming commutativity are small. This argument provides some justification for the maximum entropy approach of [6], [11], which minimizes a cost based on the covariance that satisfies (33). This justification is only valid for 
low control authority or uncertainty; otherwise, the commutativity problem could lead to erroneous stability predictions. Further details on the implications of this covariance averaging approach for maximum entropy control design can be found in [12].

\section{ACKNOWLEDGMENTS}

The authors wish to thank Nesbitt Hagood for many useful conversations, Steven Boyd for pointing out the $\mathscr{K}_{2}$ averaging result in Reference [2], and David Hyland for many useful insights into Statistical Energy Analysis and maximum entropy control theory.

\section{REFERENCES}

[1] N. W. Hagood, "Cost averaging techniques for robust control of parametrically uncertain systems," presented at ALAA Guidance, Navigation Contr. Conf., Aug. 1991, AIAA Paper 91-2605.

[2] S. P. Boyd and C. H. Barratt, Linear Controller Design: Limits of Performance. Englewood Cliffs, NJ: Prentice-Hall, 1991.

[3] E. F. Crawley, M. S. Barlow, and M. C. van Schoor, "Variation in the modal parameters of space structures," in AIAA Struc., Struc. Dyn., Materials Conf., Apr. 1992.

[4] K. B. Lazarus and E. F. Crawley, "Multivariable high-authority control of plate-like structures," in ALAA Struc., Struc. Dyn., Materials Conf., Apr. 1992.

[5] R. Lyon, Statistical Energy Analysis of Dynamical Systems: Theory and Applications. Cambridge, MA: MIT Press, 1975.

[6] D. C. Hyland, "Maximum entropy stochastic approach to controller design for uncertain structural systems," in Proc. Amer. Contr. Conf., June 1982, pp. 680-688.

[7] R. R. Mohler and W. J. Kolodziej, "An overview of stochastic bilinear control processes," IEEE Trans. Syst., Man, Cybem., vol. SMC-10, pp. 913-918, 1980.

[8] A. Graham, Kronecker Products and Matrix Calculus: With Applications. New York: Ellis Horwood Limited, 1981

[9] R. Bellman, Introduction to Matrix Analysis. New York: McGrawHill, 1970.

[10] W. F. Donoghue Jr., Distributions and Fourier Transforms. New York: Academic, 1969

[11] D. S. Bernstein and D. C. Hyland, "The optimal projection approach to robust, fixed-structure control design," in Mechanics and Control of Space Structures, J. L. Junkins, Ed., pp. 237-293. Washington, DC: AIAA, 1990.

[12] S. R. Hall, D. G. MacMartin, and D. S. Bernstein, "Covariance averaging in the analysis of uncertain systems," in Proc. IEEE Conf. Decision Contr., Dec. 1992

\section{Some Results on Minimum Magnitude Regulated Response}

Mark E. Halpern and Robin D. Hill

Abstract - In this note, analytical results are obtained for the minimum peak tracking error magnitude achievable by some finite settling time control systems in response to a step reference input. The limits of these results as the settling time approaches infinity are also obtained. These are of interest since they represent performance bounds which apply for any finite order LTI controller of a given configuration (i.e.,

Manuscript received May 31, 1991; revised January 3, 1992.

M. E. Halpem is with the Aeronautical Research Laboratory, Defence Science and Technology Organisation, Department of Defence, Australia.

R. D. Hill is with the Department of Mathematics, Royal Melbourne Institute of Technology, Melbourne, Australia.

IEEE Log Number 9212871. one-parameter or two-parameter). These limiting results have been previously given only as the numerical solution to be obtained from an infinite linear program.

The systems considered are one-parameter discrete-time SISO where the plant has one unstable pole and one nonminimum phase zero.

The result for a two-parameter compensator for plants with one nonminimum phase zero is also presented.

\section{INTRODUCTION}

The design of control systems which minimize some time domain measure of tracking error in response to an applied reference input is an important practical problem. Much of the work in this area has focussed on the minimization of a sum of squares of the tracking error over some finite or infinite time horizon. This approach may still result in some undesirably large errors, so it is of interest to consider the problem of making the largest error as small as possible.

Dahleh and Pearson [1] have examined the problem of designing a one-parameter (unity feedback) compensator which minimizes the peak magnitude of the tracking error in response to a specified input for SISO discrete-time systems.

This involved using minimum norm duality results from functional analysis to reformulate the minimization problem as its dual maximization. At optimality, the objective functions of these two problems attain the same value, that of the minimum peak error magnitude, which we call $J^{\#}$.

This dual maximization has an infinite number of constraints most of which are active. It follows for this problem, that an error sequence with a peak magnitude of $J^{\#}$ is not, in general, achievable using a finite order linear controller.

In [1], it was shown that carrying out the dual maximization subject to only the first $N+1$ dual constraints gives the minimum peak magnitude, which we denote by $J(N)$, achieveable by a dead-beat system with an error sequence duration of $N+1$ samples. It was also shown that $\lim _{N \rightarrow \infty} J(N)=J^{*}$, and a controller design technique which uses solutions to this "truncated" maximization was proposed.

The truncated dual maximization is a finite linear program (FLP), which is solved numerically in [1] to obtain the value of the achievable peak error magnitude, $J(N)$. The error sequence and compensator which achieve this may then be obtained.

Without truncation, the maximization is an infinite linear program (ILP), with an infinite number of variables and an infinite number of active constraints.

Considering the same minimum peak error magnitude problem, Moore and Bhattacharya [2] have proposed a design approach which produces an overparametrized pole placement controller, where the overparametrization is used to allow the minimization of the error magnitude for a specified closed-loop pole set. This is done by numerically solving a FLP.

In the work presented here, some analytical results for the error magnitudes, $J(N)$, achievable with finite order dead-beat controllers, and also for the optimal error magnitude, $J^{\#}$, are obtained for the tracking of a step reference. Results are given for plants with one nonminimum phase zero and one unstable pole in a one-parameter compensator system. Results are also given for plants with one nonminimum phase zero and any number of unstable poles in a two-parameter system. These results are obtained from the special structure of the dual maximizations derived in [1] and, as in that work, apply irrespective of the number of stable poles and zeros in the plant, since they are assumed to be cancelled by the controller. 